\title{
Briarane Derivatives from the Gorgonian Coral Junceella fragilis
}

\author{
Ping-Jyun Sung, ${ }^{*, a}$ Tung-Yung Fan, ${ }^{a}$ Lee-Shing Fang, ${ }^{a}$ Shwu-Li Wu,,${ }^{b, c}$ Jan-Jung LI, ${ }^{a}$ \\ Ming-Chyuan CHEN, ${ }^{a}$ Ying-Min Cheng, ${ }^{a}$ and Guey-Horng WANG ${ }^{d}$ \\ ${ }^{a}$ National Museum of Marine Biology and Aquarium; 2 Houwan Road, Checheng, Pingtung 944, Taiwan, R.O.C.: \\ ${ }^{b}$ Department of General Studies, National Kaohsiung Institute of Marine Technology; Kaohsiung 811, Taiwan, R.O.C.: \\ ${ }^{c}$ Department of Marine Resources, National Sun Yat-Sen University; Kaohsiung 804, Taiwan, R.O.C.: and ${ }^{d}$ Center for \\ General Education, Hsing-Kuo University; Tainan 709, Taiwan, R.O.C. \\ Received June 26, 2003; accepted August 6, 2003
}

\begin{abstract}
A new trihydroxyl briarane-type diterpenoid, junceellolide $H$ (1), along with two known compounds, praelolide (2) and junceellin (3), have been isolated from the gorgonian coral Junceella fragilis. The structure, including the relative configuration of the new diterpenoid 1, was elucidated by extensive spectroscopic methods.
\end{abstract}

Key words Junceella fragilis; junceellolide; praelolide; junceellin; gorgonian; briarane

In previous studies, 13 new briarane-type diterpenoids, including junceellolides $\mathrm{A}-\mathrm{G},{ }^{1,2)}(-)$-4-deacetyljunceellolide $\mathrm{D},(+)-11 \alpha, 20 \alpha$-epoxyjunceellolide $\mathrm{D},(-)-11 \alpha, 20 \alpha$ epoxy-4-deacetyljunceellolide $\quad \mathrm{D}, \quad(-)-11 \alpha, 20 \alpha$-epoxy-4deacetoxyjunceellolide $\mathrm{D},(+)$-junceellolide $\mathrm{A},{ }^{3)}$ and $9-O$ deacetylumbraculolide $\mathrm{A}$, ${ }^{4}$ ) were obtained from the gorgonian coral Junceella fragilis (Cnidaria, Anthozoa, Octocorallia, Gorgonacea, Ellisellidae), ${ }^{5-7)}$ and compounds of this type were found to possess extensive biological activities. $\left.{ }^{8}\right)$ In this paper, we report the isolation and structure determination of three briarane derivatives, including a new briarane, junceellolide $\mathrm{H}$ (1), together with two known compounds, praelolide (2) and junceellin (3), from the gorgonian Junceella fragilis collected off the Southern Taiwan coast. The structures of $\mathbf{1}-\mathbf{3}$ were elucidated by combined analyses of spectral data and by comparison with the spectral and physical data of other known compounds.

Junceellolide $\mathrm{H}$ (1) (Fig. 1) was obtained as white powder, $[\alpha]_{\mathrm{D}}^{25}-22^{\circ}\left(c=0.8, \mathrm{CHCl}_{3}\right)$. The molecular formula was determined to be $\mathrm{C}_{20} \mathrm{H}_{28} \mathrm{O}_{6}$ by high resolution (HR)-FAB-MS and NMR data. Thus, seven degrees of unsaturation were determined for $\mathbf{1}$. The IR absorptions of $\mathbf{1}$ showed the presence of hydroxyl $\left(3476 \mathrm{~cm}^{-1}\right)$ and $\gamma$-lactone $\left(1788 \mathrm{~cm}^{-1}\right)$ groups. The FAB-MS of 1 exhibited peaks at $m / z 365(\mathrm{M}+\mathrm{H})^{+}, 347$ $\left(\mathrm{M}-\mathrm{H}_{2} \mathrm{O}+\mathrm{H}\right)^{+}, 329\left(\mathrm{M}-2 \mathrm{H}_{2} \mathrm{O}+\mathrm{H}\right)^{+}$, and $311\left(\mathrm{M}-3 \mathrm{H}_{2} \mathrm{O}+\right.$ $\mathrm{H})^{+}$, also suggesting the presence of three hydroxyl groups in 1. The $1 \mathrm{D}$ and $2 \mathrm{D}$ NMR showed that 1 possesses a lactone carbonyl $\left(\delta_{\mathrm{C}} 176.2, \mathrm{~s}\right)$; two methyl substituted $(Z)$-trisubstituted olefins $\left(\delta_{\mathrm{C}} 145.9, \mathrm{~s} ; 133.6, \mathrm{~s} ; 118.4, \mathrm{~d} ; 118.1, \mathrm{~d} ; 22.8\right.$, q; $22.2, \mathrm{q} ; \delta_{\mathrm{H}} 5.38,1 \mathrm{H}$, br s; $5.24,1 \mathrm{H}, J=5.0 \mathrm{~Hz} ; 1.80,3 \mathrm{H}$, s; $1.70,3 \mathrm{H}, \mathrm{s})$; four oxymethine carbons $\left(\delta_{\mathrm{C}} 80.0, \mathrm{~d} ; 76.1, \mathrm{~d}\right.$; $74.9, \mathrm{~d} ; 65.5, \mathrm{~d} ; \delta_{\mathrm{H}} 5.58,1 \mathrm{H}, \mathrm{d}, J=5.0 \mathrm{~Hz} ; 3.68,1 \mathrm{H}$, br s; $3.59,1 \mathrm{H}$, br s; $3.40,1 \mathrm{H}, \mathrm{s})$; a methyl epoxide group $\left(\delta_{\mathrm{C}} 71.8\right.$, $\left.\mathrm{s} ; 58.8 \mathrm{~s} ; 21.7, \mathrm{q} ; \delta_{\mathrm{H}} 1.51,3 \mathrm{H}, \mathrm{s}\right)$; an aliphatic quaternary carbon $\left(\delta_{\mathrm{C}} 44.1, \mathrm{~s}\right)$; an aliphatic methine carbon $\left(\delta_{\mathrm{C}} 36.0, \mathrm{~d}\right)$; three aliphatic methylenes $\left(\delta_{\mathrm{C}} 32.4, \mathrm{t} ; 30.3, \mathrm{t} ; 26.6, \mathrm{t}\right)$; and a tertiary methyl group $\left(\delta_{\mathrm{C}} 16.5, \mathrm{q} ; \delta_{\mathrm{H}} 1.05,3 \mathrm{H}, \mathrm{s}\right)$ (Table 1$)$. By careful analysis, these data indicated that $\mathbf{1}$ is a tetracyclic briarane-type diterpenoid.

The structure and all of the assignments of ${ }^{1} \mathrm{H}$ - and ${ }^{13} \mathrm{C}$ NMR data of 1 were determined with the assistance of $2 \mathrm{D}$ NMR studies, including ${ }^{1} \mathrm{H}-{ }^{1} \mathrm{H}$ correlation spectroscopy (COSY), ${ }^{1} \mathrm{H}$-detected heteronuclear multiple quantum coher- ence (HMQC), and heteronuclear multiple bond connectivity (HMBC) experiments (Table 1, Fig. 2). From the ${ }^{1} \mathrm{H}-{ }^{1} \mathrm{H}$ COSY spectrum of $\mathbf{1}$, it was possible to establish the proton sequences from $\mathrm{H}-2$ to $\mathrm{H}_{2}-3 ; \mathrm{H}_{2}-3$ to $\mathrm{H}_{2}-4 ; \mathrm{H}-6$ to $\mathrm{H}-7$; H-9 to $\mathrm{H}-10 ; \mathrm{H}-12$ to $\mathrm{H}_{2}-13$; and $\mathrm{H}_{2}-13$ to $\mathrm{H}-14$. These data, together with the ${ }^{1} \mathrm{H}-{ }^{13} \mathrm{C}$ long-range correlations observed in an $\mathrm{HMBC}$ experiment, establish the connectivity from $\mathrm{C}-1$ to $\mathrm{C}-14$. The vinyl methyl groups attached at C-5 and C-11 were confirmed by the HMBC correlations between $\mathrm{H}_{3}-16 / \mathrm{C}$ 4, C-5, C-6; and $\mathrm{H}_{3}-20 / \mathrm{C}-10, \mathrm{C}-11, \mathrm{C}-12$. The ring-junctured $\mathrm{C}-15$ methyl group was positioned at $\mathrm{C}-1$ from the $\mathrm{HMBC}$ correlations between $\mathrm{H}_{3}-15 / \mathrm{C}-1, \mathrm{C}-2, \mathrm{C}-10$, and $\mathrm{C}-14$. Furthermore, the HMBC and ${ }^{1} \mathrm{H}-{ }^{1} \mathrm{H}$ COSY correlations also revealed that the hydroxyl groups should attach to C-2, C-9, and $\mathrm{C}-14$, respectively. These data, together with the HMBC correlations between $\mathrm{H}_{3}-18 / \mathrm{C}-8$ and $\mathrm{C}-19$, unambiguously established the molecular framework of $\mathbf{1}$.

The relative stereochemistry of $\mathbf{1}$ was deduced from a nuclear overhauser effect spectroscopy (NOESY) experiment (Fig. 3). The NOESY correlation between H-10 and H-2 indicated that these two protons are situated on the same face and were assigned as $\alpha$ protons since the C-15 methyl is the $\beta$ substituent at $\mathrm{C}-1$. $\mathrm{H}-7$ and $\mathrm{H}-14$ were found to exhibit NOE responses with $\mathrm{H}_{3}-15$, respectively, but not with $\mathrm{H}-10$, revealing the $\beta$-orientation of these two protons. Furthermore, $\mathrm{H}_{3}-18$ exhibited NOESY correlation to $\mathrm{H}-7$, indicating the $\beta$-orientation of $\mathrm{H}_{3}-18$. Also, $\mathrm{H}-9$ was found to exhibit correlations with $\mathrm{H}-7$ and $\mathrm{H}_{3}-18$, but not with $\mathrm{H}_{3}-15$. From detailed consideration of molecular models, $\mathrm{H}-9$ was found to be reasonably close to $\mathrm{H}-7$ and $\mathrm{H}_{3}-18$ when $\mathrm{H}-9$ was placed on the $\alpha$ face. Based on the above observations, the structure of $\mathbf{1}$, including the relative configuration was elucidated unambiguously. To the best of our knowledge, only

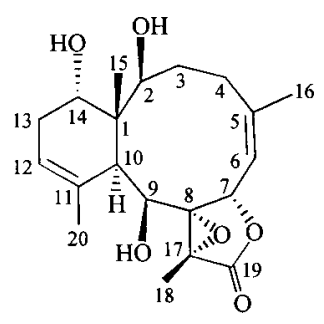

Fig. 1. The Structure of Junceellolide H (1) 
Table 1. ${ }^{1} \mathrm{H}$ - and ${ }^{13} \mathrm{C}-\mathrm{NMR}$ Data and $\mathrm{HMBC}$ and ${ }^{1} \mathrm{H}-{ }^{1} \mathrm{H}$ COSY Correlations for Junceellolide $\mathrm{H}$ (1)

\begin{tabular}{|c|c|c|c|c|}
\hline Position & ${ }^{1} \mathrm{H}$ & ${ }^{13} \mathrm{C}$ & $\mathrm{HMBC}(\mathrm{H} \rightarrow \mathrm{C})$ & ${ }^{1} \mathrm{H}-{ }^{1} \mathrm{H} \mathrm{COSY}$ \\
\hline 1 & & $44.1(\mathrm{~s})$ & $\mathrm{H}_{3}-15$ & \\
\hline 2 & $3.68(1 \mathrm{H}$, br s $)$ & 80.0 (d) & $\mathrm{H}_{2}-4, \mathrm{H}_{3}-15$ & $\mathrm{H}_{2}-3$ \\
\hline 3 & $2.62(1 \mathrm{H}, \mathrm{brs}) ; 2.19(1 \mathrm{H}, \mathrm{m})$ & $30.3(\mathrm{t})$ & $\mathrm{H}_{2}-4$ & $\mathrm{H}-2, \mathrm{H}_{2}-4$ \\
\hline 4 & $2.44(1 \mathrm{H}, \mathrm{dd}, J=14.5,10.0 \mathrm{~Hz}) ; 1.90(1 \mathrm{H}, \mathrm{m})$ & $26.6(t)$ & $\mathrm{H}_{3}-16$ & $\mathrm{H}_{2}-3$ \\
\hline 5 & & $145.9(\mathrm{~s})$ & $\mathrm{H}_{2}-4, \mathrm{H}_{3}-16$ & \\
\hline 6 & $5.24(1 \mathrm{H}, \mathrm{d}, J=5.0 \mathrm{~Hz})$ & $118.4(\mathrm{~d})$ & $\mathrm{H}_{2}-4, \mathrm{H}_{3}-16$ & $\mathrm{H}-7, \mathrm{H}_{3}-16$ \\
\hline 7 & $5.58(1 \mathrm{H}, \mathrm{d}, J=5.0 \mathrm{~Hz})$ & $76.1(\mathrm{~d})$ & $\mathrm{H}-9$ & $\mathrm{H}-6$ \\
\hline 8 & & $71.8(\mathrm{~s})$ & $\mathrm{H}_{3}-18$ & \\
\hline 9 & $3.40(1 \mathrm{H}, \mathrm{s})$ & $65.5(\mathrm{~d})$ & & $\mathrm{H}-10$ \\
\hline 10 & $2.63(1 \mathrm{H}, \mathrm{s})$ & $36.0(\mathrm{~d})$ & $\mathrm{H}-9, \mathrm{H}_{3}-15, \mathrm{H}_{3}-20$ & $\mathrm{H}-9$ \\
\hline 11 & & $133.6(\mathrm{~s})$ & $\mathrm{H}-9, \mathrm{H}_{3}-20$ & \\
\hline 12 & $5.38(1 \mathrm{H}$, br s $)$ & $118.1(\mathrm{~d})$ & $\mathrm{H}_{3}-20$ & $\mathrm{H}_{2}-13, \mathrm{H}_{3}-20$ \\
\hline 13 & $2.54(1 \mathrm{H}$, br d, $J=15.0 \mathrm{~Hz}) ; 1.99(1 \mathrm{H}, \mathrm{m})$ & $32.4(\mathrm{t})$ & & $\mathrm{H}-12, \mathrm{H}-14$ \\
\hline 14 & $3.59(1 \mathrm{H}, \mathrm{brs})$ & 74.9 (d) & $\mathrm{H}_{3}-15$ & $\mathrm{H}_{2}-13$ \\
\hline 15 & $1.05(3 \mathrm{H}, \mathrm{s})$ & $16.5(q)$ & & \\
\hline 16 & $1.80(3 \mathrm{H}, \mathrm{s})$ & $22.8(q)$ & $\mathrm{H}_{2}-4$ & H-6 \\
\hline 17 & & $58.8(\mathrm{~s})$ & & \\
\hline 18 & $1.51(3 \mathrm{H}, \mathrm{s})$ & $21.7(q)$ & & \\
\hline 19 & & $176.2(\mathrm{~s})$ & $\mathrm{H}_{3}-18$ & \\
\hline 20 & $1.70(3 \mathrm{H}, \mathrm{s})$ & $22.2(q)$ & & $\mathrm{H}-12$ \\
\hline
\end{tabular}

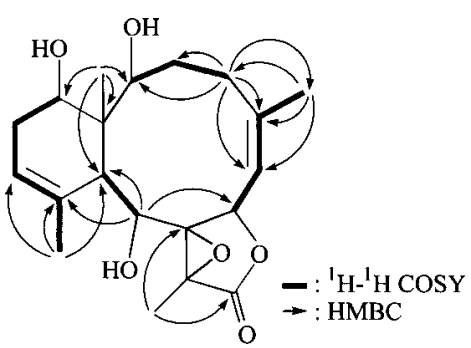

Fig. 2. Selective ${ }^{1} \mathrm{H}-{ }^{1} \mathrm{H}$ COSY and HMBC Correlations of $\mathbf{1}$

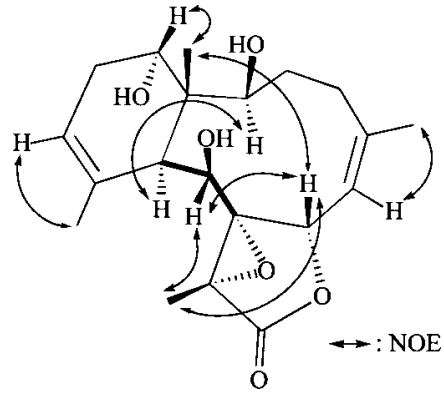

Fig. 3. Selective NOE Correlations of 1

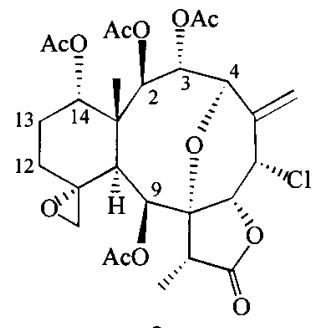

2

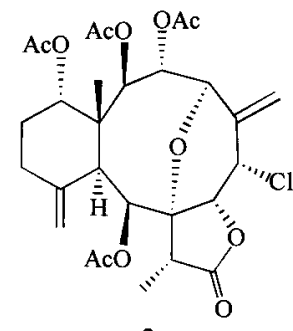

3
Fig. 4. The Structures of Diterpenoids $\mathbf{2}$ and $\mathbf{3}$

four 14-hydroxyl briarane metabolites, including junceellolide $\mathrm{G},{ }^{2)}$ gemmacolide $\mathrm{E},{ }^{9)}$ excavatolide $\mathrm{T},{ }^{10)}$ and an unnamed briarane derivative ${ }^{11)}$ were obtained from the gorgonian corals Junceella fragilis, ${ }^{2)}$ Junceella gemmacea, ${ }^{9}{ }^{9}$ Bri-
Table 2. ${ }^{13} \mathrm{C}-\mathrm{NMR}$ Data for Praelolide (2) and Junceellin (3)

\begin{tabular}{|c|c|c|c|c|}
\hline \multirow{2}{*}{ Carbon } & \multicolumn{2}{|c|}{2} & \multicolumn{2}{|c|}{3} \\
\hline & ${ }^{13} \mathrm{C}$ & ${ }^{13} \mathrm{C}^{a)}$ & ${ }^{13} \mathrm{C}$ & ${ }^{13} \mathrm{C}^{a)}$ \\
\hline 1 & $46.7(\mathrm{~s})$ & $46.9(\mathrm{~s})$ & $47.4(\mathrm{~s})$ & $47.4(\mathrm{~s})$ \\
\hline 2 & 72.8 (d) & $78.5(\mathrm{~d})$ & 72.8 (d) & 77.5 (d) \\
\hline 3 & 63.8 (d) & 74.0 (d) & 63.7 (d) & 78.8 (d) \\
\hline 4 & 78.8 (d) & $64.0(\mathrm{~d})$ & 78.8 (d) & 63.7 (d) \\
\hline 5 & $134.2(\mathrm{~s})$ & $134.4(\mathrm{~s})$ & $134.2(\mathrm{~s})$ & $134.2(\mathrm{~s})$ \\
\hline 6 & $53.8(\mathrm{~d})$ & 54.0 (d) & $53.9(\mathrm{~d})$ & $53.9(\mathrm{~d})$ \\
\hline 7 & 79.0 (d) & 79.1 (d) & 79.1 (d) & 79.1 (d) \\
\hline 8 & $82.8(\mathrm{~s})$ & $82.9(\mathrm{~s})$ & $82.7(\mathrm{~s})$ & 82.7 (s) \\
\hline 9 & 70.8 (d) & 72.9 (d) & 77.5 (d) & 74.5 (d) \\
\hline 10 & 40.9 (d) & 41.0 (d) & 44.0 (d) & 44.0 (d) \\
\hline 11 & $56.1(\mathrm{~s})$ & $56.2(\mathrm{~s})$ & $147.2(\mathrm{~s})$ & $147.2(\mathrm{~s})$ \\
\hline 12 & $29.6(\mathrm{t})$ & $24.6(t)$ & $32.6(\mathrm{t})$ & $27.5(\mathrm{t})$ \\
\hline 13 & $24.6(\mathrm{t})$ & $29.3(\mathrm{t})$ & $27.5(\mathrm{t})$ & $32.6(\mathrm{t})$ \\
\hline 14 & 73.8 (d) & $71.0(\mathrm{~d})$ & 74.5 (d) & 72.8 (d) \\
\hline 15 & $15.8(q)$ & $15.8(q)$ & $15.0(q)$ & $15.0(q)$ \\
\hline 16 & $119.5(\mathrm{t})$ & $119.4(t)$ & $119.5(\mathrm{t})$ & $119.6(\mathrm{t})$ \\
\hline 17 & 49.4 (d) & 49.5 (d) & 49.9 (d) & 49.9 (d) \\
\hline 18 & $7.2(q)$ & $7.3(q)$ & $7.1(q)$ & $7.1(q)$ \\
\hline 19 & $174.2(\mathrm{~s})$ & $174.2(\mathrm{~s})$ & $174.1(\mathrm{~s})$ & $174.2(\mathrm{~s})$ \\
\hline 20 & $51.2(\mathrm{t})$ & $51.3(\mathrm{t})$ & $111.9(\mathrm{t})$ & $111.9(\mathrm{t})$ \\
\hline \multirow[t]{4}{*}{ Acetate methyls } & $21.1(\mathrm{q})$ & $21.1(q)$ & $21.0(\mathrm{q})$ & $21.0(q)$ \\
\hline & 20.9 (q) & 20.9 (q) & $21.0(q)$ & $21.0(q)$ \\
\hline & 20.4 (q) & 20.4 (q) & 20.5 (q) & $20.5(q)$ \\
\hline & $20.3(q)$ & $20.3(\mathrm{q})$ & $20.4(q)$ & $20.4(q)$ \\
\hline \multirow[t]{4}{*}{ Acetate carbonyls } & $170.2(\mathrm{~s})$ & $170.2(\mathrm{~s})$ & $170.4(\mathrm{~s})$ & $170.4(\mathrm{~s})$ \\
\hline & $169.9(\mathrm{~s})$ & $169.9(\mathrm{~s})$ & $170.0(\mathrm{~s})$ & $170.0(\mathrm{~s})$ \\
\hline & $169.8(\mathrm{~s})$ & $169.9(\mathrm{~s})$ & $169.8(\mathrm{~s})$ & $169.8(\mathrm{~s})$ \\
\hline & $169.5(\mathrm{~s})$ & $169.6(\mathrm{~s})$ & $169.7(\mathrm{~s})$ & $169.7(\mathrm{~s})$ \\
\hline
\end{tabular}

a) The data were reported by Subrahmanyam et al., see ref. 14 .

areum excavatum, ${ }^{10)}$ and a soft coral belonging to the genus Nephthea,${ }^{11)}$ respectively.

The two known chlorinated compounds, praelolide (2) and junceellin (3) (Fig. 4), were previously identified by their physical and spectral data. Briaranes $\mathbf{2}$ and $\mathbf{3}$ were first isolated from the South China Sea gorgonian corals Plexaureides praelonga and Junceella squamata, respectively; ${ }^{12,13)}$ these two metabolites were also obtained from an Indian 
Ocean gorgonian Gorgonella umbraculum. ${ }^{14)}$ Moreover, based on detailed analyses of 1D and 2D NMR spectra, the ${ }^{13} \mathrm{C}-\mathrm{NMR}$ chemical shifts for the oxymethines C-2, C-3, C-4, C-9, and C-14 and the methylenes C-12 and C-13 of briaranes $\mathbf{2}$ and $\mathbf{3}$ were reassigned in this study (Table 2).

Although many briarane-based diterpenoids have been shown to exhibit various biological activities, ${ }^{8)}$ briaranes 1 3 were found not to be cytotoxic toward a limited panel of tumor cell lines. These cells are P-388D1 (mouse lymphoid neoplasm), DLD-1 (human colon adenocarcinoma), IMR-32 (human neuroblastoma), RPMI 7951 (human malignant melanoma), and CCRF-CEM (human T-cell acute lymphoblastic leukemia) cells.

\section{Experimental}

Melting points were determined using a FARGO apparatus and were uncorrected. Optical rotations were measured in $\mathrm{CHCl}_{3}$ with a JASCO D-370 digital polarimeter at $25^{\circ} \mathrm{C}$. Infrared spectra were measured on a JASCO 5300 FT-IR spectrometer. Mass spectral data were obtained with a VG QUATTRO GC/MS spectrometer. HR-FAB-MS was recorded on a JEOL JMS SX/SX 102A mass spectrometer. NMR spectra were recorded on a VARIAN UNITY INOVA 500 FT-NMR at $500 \mathrm{MHz}$ for ${ }^{1} \mathrm{H}$ and $125 \mathrm{MHz}$ for ${ }^{13} \mathrm{C}$, respectively, in $\mathrm{CDCl}_{3}$ using TMS as an internal standard. Silica gel (Merck, 230-400 mesh) was used for column chromatography. Precoated silica gel plates (Merck, Kieselgel $60 \mathrm{~F}_{254}$ ) were used for analytical TLC. All solvents used were either freshly distilled or of analytical grade.

Animal Material Specimens of J. fragilis were collected by hand using scuba gear off the Southern Taiwan coast in Dec. 2002, at a depth of $-10 \mathrm{~m}$. Living reference specimens are being maintained in the authors' tanks and the voucher specimen was deposited in the National Museum of Marine Biology and Aquarium. (specimen no. TWGC-003). This organism was identified from descriptions. $\left.{ }^{5-7}\right)$

Extraction and Isolation The organism ( $780 \mathrm{~g}$ ) was collected and freeze-dried. The freeze-dried material $(557 \mathrm{~g})$ was minced and extracted with EtOAc $(5 \times 500 \mathrm{ml})$ for $120 \mathrm{~h}$ at $25^{\circ} \mathrm{C}$. The organic extract $(11.1 \mathrm{~g})$ was separated by silica gel column chromatography using hexanes and hexanes-EtOAc mixtures of increasing polarity. Briarane 1 was eluted with hexanes-EtOAc ( $2: 1), \mathbf{2}$ with hexanes-EtOAc $(4: 1)$, and 3 with hexanes-EtOAc $(5: 1)$.

Junceellolide $\mathrm{H}(\mathbf{1})$ : White powder $(7.1 \mathrm{mg}) ; \mathrm{mp} 207-209^{\circ} \mathrm{C} ;[\alpha]_{\mathrm{D}}^{25}$ $-22^{\circ}\left(c=0.8, \mathrm{CHCl}_{3}\right)$; IR (neat) $\mathrm{cm}^{-1} 3476,1788 ;{ }^{1} \mathrm{H}-$ and ${ }^{13} \mathrm{C}-\mathrm{NMR}$ $\left(\mathrm{CDCl}_{3}\right)$, see Table 1; FAB-MS $m / z$ : $365\left(\mathrm{M}^{+}+\mathrm{H}\right), 347,329,311,289,271$, 255. HR-FAB-MS $m / z$ : 365.1957 (Calcd for $\mathrm{C}_{20} \mathrm{H}_{28} \mathrm{O}_{6}+\mathrm{H}: 365.1965$ ).

Praelolide (2): White powder $(44.1 \mathrm{mg}) ; \mathrm{mp} 267-269^{\circ} \mathrm{C} ;[\alpha]_{\mathrm{D}}^{25}-26^{\circ}$ $\left(c=2.4, \mathrm{CHCl}_{3}\right)$; IR (neat) $\mathrm{cm}^{-1} 1794,1744,1606,932 ;{ }^{1} \mathrm{H}-\mathrm{NMR}(500$ $\left.\mathrm{MHz} \mathrm{CDCl}_{3}\right) \delta 6.19(1 \mathrm{H}, \mathrm{dd}, J=11.0,7.0 \mathrm{~Hz}, \mathrm{H}-3), 5.59(1 \mathrm{H}, \mathrm{s}, \mathrm{H}-9), 5.56$ $(1 \mathrm{H}, \mathrm{d}, J=2.0 \mathrm{~Hz}, \mathrm{H}-16 \mathrm{a}), 5.39(1 \mathrm{H}, \mathrm{d}, J=7.0 \mathrm{~Hz}, \mathrm{H}-2), 5.35(1 \mathrm{H}, \mathrm{d}$, $J=2.0 \mathrm{~Hz}, \mathrm{H}-16 \mathrm{~b}), 4.99(1 \mathrm{H}$, br t, $J=2.5 \mathrm{~Hz}, \mathrm{H}-14), 4.97(1 \mathrm{H}, \mathrm{d}, J=3.0 \mathrm{~Hz}$, H-6), $4.46(1 \mathrm{H}, \mathrm{d}, J=11.0 \mathrm{~Hz}, \mathrm{H}-4), 4.40(1 \mathrm{H}, \mathrm{d}, J=3.0 \mathrm{~Hz}, \mathrm{H}-7), 2.83(1 \mathrm{H}$, s, H-10), 2.81 (1H, q, $J=7.0 \mathrm{~Hz}, \mathrm{H}-17), 2.66(1 \mathrm{H}, \mathrm{d}, J=3.5 \mathrm{~Hz}, \mathrm{H}-20 \mathrm{a}), 2.45$ (1H, d, J=3.5 Hz, H-20b), 2.31 (3H, s, acetate methyl), 2.19 (1H, m, H-12), $2.08(3 \mathrm{H}, \mathrm{s}$, acetate methyl), $2.06(3 \mathrm{H}, \mathrm{s}$, acetate methyl $), 2.00(3 \mathrm{H}, \mathrm{s}$, ac- etate methyl), $1.91(1 \mathrm{H}, \mathrm{m}, \mathrm{H}-13), 1.88\left(1 \mathrm{H}, \mathrm{m}, \mathrm{H}-13^{\prime}\right), 1.33(3 \mathrm{H}, \mathrm{d}$, $\left.J=7.0 \mathrm{~Hz}, \mathrm{H}_{3}-18\right), 1.29\left(1 \mathrm{H}, \mathrm{m}, \mathrm{H}-12^{\prime}\right)$, and $1.24\left(3 \mathrm{H}, \mathrm{s}, \mathrm{H}_{3}-15\right) ;{ }^{13} \mathrm{C}-\mathrm{NMR}$ $\left(125 \mathrm{MHz}, \mathrm{CDCl}_{3}\right)$ : Table 2; electron impact (EI)-MS $m / z: 600\left(\mathrm{M}^{+}+2\right), 598$ $\left(\mathrm{M}^{+}\right), 243$. The related physical ( $\mathrm{mp}$ and optical rotation value) and spectral (IR, ${ }^{1} \mathrm{H}-\mathrm{NMR}, \mathrm{MS}$ ) data of $\mathbf{2}$ are in full agreement with those reported previously. $^{12,14)}$

Junceellin (3): White powder $(15.1 \mathrm{mg}) ; \mathrm{mp} 271-272{ }^{\circ} \mathrm{C} ;[\alpha]_{\mathrm{D}}^{25}-10^{\circ}$ $\left(c=1.8, \mathrm{CHCl}_{3}\right) ; \mathrm{IR}$ (neat) $\mathrm{cm}^{-1} 1796,1744,1608,930 ;{ }^{1} \mathrm{H}-\mathrm{NMR}$ (500 $\left.\mathrm{MHz} \mathrm{CDCl}_{3}\right) \delta 6.14(1 \mathrm{H}, \mathrm{dd}, J=11.0,6.5 \mathrm{~Hz}, \mathrm{H}-3), 5.94(1 \mathrm{H}, \mathrm{s}, \mathrm{H}-9), 5.57$ $(1 \mathrm{H}, \mathrm{d}, J=2.0 \mathrm{~Hz}, \mathrm{H}-16 \mathrm{a}), 5.43(1 \mathrm{H}, \mathrm{d}, J=6.5 \mathrm{~Hz}, \mathrm{H}-2), 5.36(1 \mathrm{H}, \mathrm{d}$, $J=2.0 \mathrm{~Hz}, \mathrm{H}-16 \mathrm{~b}), 5.10$ (1H, s, H-20a), 5.02 (1H, d, $J=3.0 \mathrm{~Hz}, \mathrm{H}-6), 4.97$ $(1 \mathrm{H}$, br t, $J=3.0 \mathrm{~Hz}, \mathrm{H}-14), 4.77(1 \mathrm{H}, \mathrm{s}, \mathrm{H}-20 \mathrm{~b}), 4.51(1 \mathrm{H}, \mathrm{d}, J=3.0 \mathrm{~Hz}, \mathrm{H}-$ 7), $4.48(1 \mathrm{H}, \mathrm{d}, J=11.0 \mathrm{~Hz}, \mathrm{H}-4), 3.11(1 \mathrm{H}, \mathrm{s}, \mathrm{H}-10), 2.78(1 \mathrm{H}, \mathrm{q}, J=7.0 \mathrm{~Hz}$, H-17), $2.32(3 \mathrm{H}, \mathrm{s}$, acetate methyl), $2.31(1 \mathrm{H}, \mathrm{m}, \mathrm{H}-12), 2.25(1 \mathrm{H}, \mathrm{m}, \mathrm{H}-$ $\left.12^{\prime}\right), 2.07(3 \mathrm{H}, \mathrm{s}$, acetate methyl), $2.05(3 \mathrm{H}, \mathrm{s}$, acetate methyl), $2.00(3 \mathrm{H}, \mathrm{s}$, acetate methyl), $1.82(1 \mathrm{H}, \mathrm{m}, \mathrm{H}-13), 1.70\left(1 \mathrm{H}, \mathrm{m}, \mathrm{H}-13^{\prime}\right), 1.29(3 \mathrm{H}, \mathrm{d}$, $\left.J=7.0 \mathrm{~Hz}, \mathrm{H}_{3}-18\right)$, and $1.12\left(3 \mathrm{H}, \mathrm{s}, \mathrm{H}_{3}-15\right)$; ${ }^{13} \mathrm{C}-\mathrm{NMR}\left(125 \mathrm{MHz}, \mathrm{CDCl}_{3}\right)$ : Table 2; EI-MS $m / z$ : $584\left(\mathrm{M}^{+}+2\right), 582\left(\mathrm{M}^{+}\right), 243$. The related physical (mp and optical rotation value) and spectral (IR, $\left.{ }^{1} \mathrm{H}-\mathrm{NMR}, \mathrm{MS}\right)$ data of $\mathbf{3}$ are in full agreement with those reported previously. ${ }^{13,14}$

Cytotoxicity Testing The cytotoxicity of tested compounds $\mathbf{1}-\mathbf{3}$ was assayed with a modification of the MTT [3-(4,5-dimethylthiazol-2-yl)-2,5diphenyltetrazolium bromide] colorimetric method. Cytotoxicity assays were carried out according to the procedures described previously. ${ }^{15}$ )

Acknowledgments This research work was supported by grants from the National Science Council of the Republic of China (Contract no. NSC 91-2320-B-291-001 and NSC 92-2323-B-291-001) awarded to P.-J. Sung.

\section{References}

1) Shin J., Park M., Fenical W., Tetrahedron, 45, 1633-1638 (1989).

2) Sung P.-J., Wu S.-L., Fang H.-J., Chiang M. Y., Wu J.-Y., Fang L.-S., Sheu J.-H., J. Nat. Prod., 63, 1483-1487 (2000).

3) García M., Rodríguez J., Jiménez C., J. Nat. Prod., 62, 257-260 (1999).

4) Sung P.-J., Fan T.-Y., Heterocycles, 60, 1199-1202 (2003).

5) Bayer F. M., Proc. Biol. Soc. Wash., 94, 902-947 (1981).

6) Chen C.-C., Chang K.-H., Bull. Inst. Zool., Academia Sinica, 30, 149-182 (1991).

7) Bayer F. M., Grasshoff M., Senckenbergiana Biol., 74, $21-45$ (1994).

8) Sung P.-J., Sheu J.-H., Xu J.-P., Heterocycles, 57, 535-579 (2002).

9) He H.-Y., Faulkner D. J., Tetrahedron, 47, 3271-3280 (1991).

10) Neve J. E., McCool B. J., Bowden B. F., Aust. J. Chem., 52, 359-366 (1999).

11) Anjaneyulu A. S. R., Krishna Murthy M. V. R., Indian J. Chem., 39B, $42-56$ (2000).

12) Luo Y., Long K., Fang Z., Zhongshan Daxue Xuebao, Ziran Kexueban, 1, 83-92 (1983).

13) Lin Y., Long K., Zhongshan Daxue Xuebao, Ziran Kexueban, 2, 46 51 (1983).

14) Subrahmanyam C., Kulatheeswaran R., Ward R. S., J. Nat. Prod., 61, 1120-1122 (1998).

15) Alley M. C., Acudiero D. A., Monks A., Hursey M. L., Czerwinski M. J., Fine D. L., Abbott B. J., Mayo J. G., Shoemark R. H., Boyd M. R., Cancer Res., 48, 589-601 (1988). 